proportion of arm and leg casts. However, lower limb casts were more likely to need reinforcing (13 out of 17). All plasters that were trimmed were on the upper limb. Two plaster technicians were responsible for $52 \%$ of the casts applied and $41 \%$ of those requiring alteration. No plaster was altered because of signs or symptoms of neurovascular compression. Of the 159 who had no change made at their attendance, six patients returned prior to their clinic appointments for alterations, three requested a replacement having removed their own forearm slabs after a week. Two plasters were replaced when broken and one was trimmed. Only two patients of the 26 who did not attend for a plaster check returned with problems, one requiring replacement of a broken cast and one who returned with persistent pain 3 days after manipulation of her Colles fracture but who required no alteration.

The postal questionnaire had a $72 \%$ response rate and of these, $31 \%$ remembered being given additional advice - however, only six of the $\mathbf{3 0}$ who reported subsequent problems with the cast had returned for changes to be made. Returning for the check was stated as inconvenient by $16 \%$ and worthwhile by $82 \%$.

In view of the $24 \%$ alteration rate it will remain this department's practice to offer a plaster check to all those having a plaster applied as, unfortunately, patients from all groups return with a plethora of problems.

G. RIDING, M. EDGELL \& M. JAMES Accident and Emergency Department, Royal Preston Hospital, Sharoe Green Lane North, Fulwood, Preston

\section{Is rapid myoglobin measurement of diagnostic value in the emergency presentation of non-traumatic chest pain?}

As there is an established treatment for acute myocardial infarction (AMI) in the form of thrombolytic therapy, ${ }^{1,2}$ early diagnosis is essential. In cases presenting to the accident and emergency (A\&E) department, the diagnosis of AMI is not always immediately obvious. A rapid, sensitive and specific test for the early diagnosis of AMI could prove useful as a guide to appropriate referral and subsequent therapy.

Myoglobin is known to be released up to four hours earlier than both creatine kinase (CK) and CK-MB following AMI, with peak levels being achieved up to 14 hours earlier. ${ }^{3}$ There is now available an immunoturbidimetric method to measure the myoglobin concentration in serum or plasma within minutes. ${ }^{4}$ We undertook to evaluate, retrospectively, the usefulness of this assay in the acute situation.

The study was carried out on patients presenting to the A\&E Department of the Western Infirmary, Glasgow, with non-traumatic chest pain. The patients were either general practitioner-referred, or self-referred.

A total of 136 patients presenting with chest pain were studied: 73 males and 63 females. The age range was 16 to 82 years, the median age was 58 years. The A\&E officer attending the patient took the blood sample for analysis as soon as practicable (within $1 \mathrm{~h}$ of presentation). Retrospective analysis was carried out on each sample for myoglobin, total CK and CK-MB if the CK was found to be greater than $100 \mu \mathrm{gL}^{-1}$.

Myoglobin concentration was determined using the Behring Turbiquant Myoglobin assay system (Behringwerke AG Diagnostica, Marburg, Germany) as recommended by the manufacturer. This assay, which may be used on- or off-site, is a rapid, particle enhanced assay based on the reaction between the myoglobin present in the sample and anti-myoglobin antibodies coupled to polystyrene particles. The resultant increase in turbidity is measured photometrically. The measuring range of the assay is $50-650 \mu \mathrm{g} \mathrm{L}^{-1}$.

Of the 136 patients involved in the study, 32 patients were discharged directly from the A\&E department without subsequent hospital follow-up. Although none of the patients discharged directly had either a raised serum myoglobin concentration or a raised CK-MB ratio, or were subsequently readmitted to the Western Infirmary with a related complaint, this does not completely exclude the possibility of AMI in any of those patients.

A total of 104 patients were referred for admission and further investigation either to the coronary care unit, or to the general medical wards. Of the patients admitted, 27 were diagnosed as having sustained an AMI either at presentation, on clinical and electrocardiographic evidence, or on subsequent investigation. In all cases, AMI was confirmed by a raised CK-MB ratio (>5.9). The remaining 77 patients had an AMI excluded using the same criteria.

Myoglobin concentration, on admission, was found to be significantly elevated $\left(>100 \mu \mathrm{gL}^{-1}\right)$ in 14 of the 27 AMI patients as compared with 11 patients with a raised total CK and CK-MB ratio. 
Two patients with a myoglobin concentration within the normal range on admission, had a raised total CK and CK-MB ratio. Both patients presented to the A\&E department more than $24 \mathrm{~h}$ from the onset of symptoms.

One of the main reasons for early diagnosis of AMI is to allow the earliest possible administration of thrombolytic therapy. We therefore reviewed the number of patients receiving this therapy and the reasons for non-administration. Of the $27 \mathrm{AMI}$ patients, 11 were given thrombolytic therapy in the form of intravenous streptokinase. Ten patients did not receive therapy as, based on the available clinical and electrocardiographic evidence at presentation, the diagnosis was thought to be ischaemic chest pain short of AMI. Five patients were over 70 years of age and one patient was already on warfarin therapy.

Reviewing the 16 AMI patients who did not receive thrombolytic therapy revealed that four of the 10 patients thought to have ischaemic chest pain short of infarction would have been accurately diagnosed on admission if the myoglobin concentration result had been available. Potentially, another four AMI patients may have benefited from thrombolytic therapy.

In trying to make an accurate diagnosis of $\mathrm{AMI}$ in the A\&E department, it is well recognized that to rely on electrocardiographic changes alone could miss up to $50 \%$ of cases. Single or serial measurements of CK-MB are of limited value in the first four hours after the onset of symptoms. ${ }^{5}$ The recognized earlier release of myoglobin following $\mathrm{AMI}$ coupled with a rapid and sensitive test for its detection, makes it potentially more useful. ${ }^{6}$

Our data, on the patients subsequently admitted from the A\&E department, show myoglobin concentration measurement on presentation to have a sensitivity of $51.9 \%$ as compared with a sensitivity of $40.7 \%$ for CK-MB in the diagnosis of AMI. Obviously both sensitivities are too low to be of diagnostic value in the acute situation.

The important question is why 13 patients subsequently diagnosed as having sustained an AMI had a normal myoglobin concentration on admission. Two of the patients presented more than $24 \mathrm{~h}$ from the onset of symptoms: at a time when the myoglobin concentration, even if previously elevated, could have returned to normal. ${ }^{7} A$ further four patients presented less than $2 \mathrm{~h}$ from the onset of symptoms: too early to see a diagnostic rise in myoglobin concentration. ${ }^{3}$ The remaining false negative tests patient variability in the perception of pain, and the fact that the onset of chest pain does not necessarily prove the onset of critical or permanent occlusion of the coronary artery. We found a poor correlation between the time elapsed from patient symptom onset to blood sampling, the results found on analysis, and the known timing of release of biochemical markers following AMI.

In this study, myoglobin concentration measurement is more sensitive than total CK or CK-MB for the diagnosis of $\mathrm{AMI}$ in patients presenting with non-traumatic chest pain to the A\&E department who are subsequently admitted. However diagnostic sensitivity only reached $51.9 \%$ in this patient group, partly as a result of early and late presentation of patients.

Myoglobin concentration measurements may be of diagnostic value in equivocal cases, but we have found no evidence to support the routine, uncontrolled, immediate measurement of myoglobin concentration in all patients presenting to an A\&E department with non-traumatic chest pain.

\section{ACKNOWLEDGEMENTS}

The authors would like to thank the A\&E Officers of the A\&E department of the Western Infirmary, Glasgow for their cooperation in taking the patient blood samples and Behring for their help in purchasing the reagents used in the study.

\section{W.S. KILPATRICK, ${ }^{1}$ R.J. SPOONER, ${ }^{1}$} P. GRANT ${ }^{2}$ \& W. TULLETT ${ }^{2}$

Departments of ${ }^{1}$ Pathological Biochemistry and ${ }^{2}$ Accident and Emergency Medicine, Western Infirmary, Dumbarton Road, Glasgow

\section{REFERENCES}

1. GISSI (Italian Group for the Study of Streptokinase in Myocardial Infarction) (1986) Effectiveness of intravenous thrombolytic treatment in acute myocardial infarction. Lancet 1, 397-401.

2. ISIS-2 (Second International Study of Infarct Survival) Collaborative Group. (1988) Randomised trial of intravenous streptokinase, oral aspirin, both or neither among 17,187 cases of suspected acute myocardial infarction. Lancet 2, 349-360.

3. Grenadier E., Keidar S., Kahana L., Alpan G., Marmur A. \& Palant A. (1983) The roles of serum myoglobin, total CPK, and CK-MB isoenzyme in the acute phase of myocardial infarction. American Heart Journal 105, 408-416.

4. Delanghe J., Chapelle J.-P., El Allaf M. \& De Buyzere M. 
(1991) Quantitative turbidimetric assay for determining myoglobin evaluated. Annals of Clinical Biochemistry 28, 474-479.

5. Collinson P.O., Rosalki S.B., Kuwana T., Garratt H.M., Ramhamadamy E.M., Baird I.M. \& Greenwood T.W. (1992) Early diagnosis of acute myocardial infarction by CK-MB mass measurements. Annals of Clinical Biochemistry 29, 43-47.

6. Kilpatrick W.S., Wosornu D., McGuinness J.B. \& Glen A.C.A. (1993) Early diagnosis of acute myocardial infarction: CK-MB and myoglobin compared. Annals of Clinical Biochemistry 30, 435-438.

7. Drexel H., Dworzak E., Kirchmair W., Milz M.M., Puschendorf B. \& Dienstl F. (1983) Myoglobinaemia in the early phase of acute myocardial infarction. American Heart Journal 105, 642-651.

\section{An unexpected foreign body: a case of external opthalmomyiasis}

An 11-year-old boy recently presented to the Accident and Emergency (A\&E) Department of the Birmingham and Midland Eye Hospital complaining that a fly had hit his right eye and felt as if it was still in the eye. This had occurred the previous day, at the end of a 2-week holiday in Rhodes. On eversion of the upper lid four moving pin head sized larvae were seen on the inflamed conjunctiva (Fig. 1). The larvae were removed with a cotton bud and the boy was given chloramphenicol drops to treat any secondary infection. The discomfort had settled on review 2 days later.

The larvae were identified by Dr Anderson of The University of Birmingham, as the first instar larvae of Oestrus ovis, the sheep nostril fly. Female Oestrus ovus flies are larviparous, usually squirting their larvae up sheep nostrils whilst in flight. Occasionally their larvae go astray.

The invasion of tissues by larvae is called myiasis. This particular case is an example of external ophthalmomyiasis. The larvae can burrow into the eyelid, conjunctiva and lacrimal ducts using both the spines on their eleven body segments and their large oral hooks. Rarely, the larvae may penetrate the eyeball causing blindness. ${ }^{1}$

Oestrus ovis are found in North Africa, Asia and the Mediterranean. $^{2}$ Although, three cases are thought to have occurred in England. ${ }^{3}$ Previously only one case has been reported as being 'imported' to England. ${ }^{4}$ Bearing in mind the prevalence of travel to the Mediterranean nowadays, such conditions should be looked out for and one should remember to check under the eyelid.

\section{S.J. TALKS}

Birmingham and Midland Eye Hospital, Churgh Street, Birmingham B3 2NS

\section{REFERENCES}

1. Rakusin W. (1970) Ocular myiasis interna caused by the sheep nasal bot fly (Oestrus ovis). South African Medical Journal 44, 1155-1162.

2. Reid A.M. (1972) Ophthalmology in the tropics. In: Manson's Tropical Diseases. (eds C. Wilcocks \& P.E.C. Manson-Bahr), pp. 829-890. Bailliere Tindall, London.

3. Stevens J.D., McCartney A.C.E. \& Howes R. (1991) Oestrus ovis ophthalmomyiasis acquired in the U.K.: case report and scanning electron microscopic study. British Journal of Ophthalmology 75, 702-703.

4. Wong D. (1982) External ophthalmomyiasis caused by the sheep bot Oestrus ovis L. British Journal of Ophthalmology 66, 786-787.

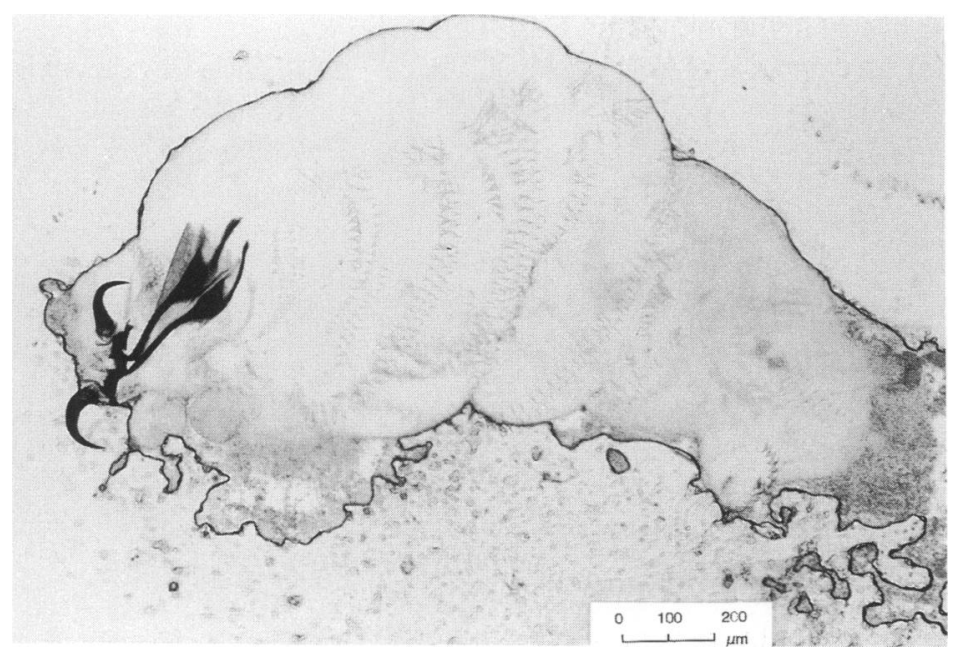

Fig. 1. One of the Oestrus ovis following removal from the patient's eye. 